\title{
CONSULTÓRIO FARMACÊUTICO EM DROGARIA
}

\author{
DRUG STORE PHARMACEUTICAL OFFICE
}

\author{
Joyce de Mello Sarmento Marques ${ }^{1}$ \\ Alex Sandro Rodrigues Baiense ${ }^{2}$
}

RESUMO: A humanidade convive com muitos males, o que muitas vezes necessita de medicação, por isso o farmacêutico é de fundamental relevância quanto ao acompanhamento farmacoterapêutico, uma vez que o paciente necessita de cuidados e orientação quanto ao uso racional de medicamentos e hábitos de vida adequados para o controle de suas doenças. Diante disso, a prescrição farmacêutica através de consultório é um momento histórico para a profissão trazendo a tona o papel do farmacêutico para toda a população, o farmacêutico não é mais um indicador de medicamentos é um profissional que auxilia na expectativa de tratamento.

Palavras-chave: Consultório Farmacêutico. Drogarias. Atenção Farmacêutica. Cuidado ao Paciente.

ABSTRACT: Humanity lives with many males, which often require medication, so the pharmacist is essential in terms of pharmacotherapeutic monitoring, since the patient needs care and guidance on the rational use of medications and appropriate lifestyle habits for the control of their diseases. Therefore, pharmaceutical prescription through the office is a historic moment for the profession, bringing out the role of the pharmacist for the entire population, the pharmacist is no longer an indicator of medications, he is a professional who helps in the expectation of treatment.

Keywords: Pharmaceutical Office. Drugstores. Pharmaceutical attention. Patient Care.

\section{INTRODUÇÃO}

O farmacêutico desde as antigas "boticas", com seus conhecimentos de química, botânica entre outros sempre contribui com a pesquisa, manipulação e dispensação de produtos farmacêuticos garantindo a qualidade dos produtos, e assegurando que os pacientes saibam sobre o uso correto dos medicamentos incluindo os medicamentos de venda livre (HEPLER, 1990).

\footnotetext{
${ }^{\mathrm{I}}$ UNIG - Universidade Iguaçu, Curso de Graduação em Farmácia, Nova Iguaçu- RJ, Brasil

${ }^{2}$ Orientador: Nova Iguaçu- RJ.
} 
O papel do farmacêutico sempre foi importante, e atualmente, com o novo modelo assistencial, onde a ênfase é a atenção primária à saúde, o farmacêutico torna-se, na maioria das vezes, o último profissional a ter contato direto com o paciente (CFF, 2016).

As atividades clínicas do farmacêutico podem ser realizadas em todos os níveis de atenção à saúde, exigindo-se para tal o perfil adequado do profissional. Nesse contexto, o farmacêutico deve atuar no cuidado direto ao paciente através de orientações quanto ao uso racional de medicamentos e de outras tecnologias em saúde, otimização da farmacoterapia, quando necessário, e promover a educação em saúde, além de poder realizar outras atribuições que lhe são conferidas (BRASIL, 2013).

O farmacêutico é o profissional habilitado a orientar o usuário quanto ao uso correto de medicamento, bem como, aos riscos envolvidos na terapia, tendo como base as necessidades de saúde do paciente. Desde agosto de 2013, o papel do farmacêutico ganhou novas prerrogativas, com a regulamentação da prescrição farmacêutica (CFF, 2016).

Em uma sala, o consultório farmacêutico, é atribuído ao profissional a realização da consulta farmacêutica, sendo garantido nesse espaço um atendimento com privacidade ao paciente, possibilitando assim que o farmacêutico atue mediante exercício legal da profissão (BRASIL, 2013).

Atualmente o farmacêutico pode fazer a prescrição farmacêutica utilizando critérios clínicos de semiologia e anamnese realizando indicações com segurança assim, aumentando significativamente seu poder de atuação, na qual as farmácias magistrais poderão manipular prescrições de farmacêuticos que atendam em drogarias e farmácias, bem como as farmácias magistrais, ambulatórios, farmácias comunitárias, entre outros estabelecimentos farmacêuticos (CRF-SP, 2014).

\section{OBJETIVOS}

\section{I Objetivo Geral}

Identificar os serviços de atenção farmacêutica prestados no consultório farmacêutico. 


\subsection{Objetivos Específicos}

- Exemplificar o que é um consultório farmacêutico;

- Identificar as obrigações do farmacêutico no consultório;

- Listar os serviços prestados no consultório Farmacêutico;

- Mencionar como é feita a implantação de um consultório farmacêutico;

- Entender o papel do farmacêutico nas intervenções medicamentosas.

\section{METODOLOGIA}

A metodologia utilizada foi de levantamento bibliográfico, utilizando as palavras-chave: Consultório Farmacêutico; Drogarias; Atenção Farmacêutica; Cuidado ao Paciente. A ferramenta de busca foram LILACS (Literatura LatinoAmericana e do Caribe em Ciências da Saúde), SciELO (Scientific Electronic Library Online) e MEDLINE (Medical Literature Analysis and Retrievel System Online), artigos, revistas e livros impressos no período da 2010 a 2021. Foram incluídos artigos originais de pesquisa qualitativa e bibliográfica. Os critérios de exclusão: artigos que não tinham a temática escolhida.

\section{JUSTIFICATIVA}

Justifica-se a escolha do tema, por muitos desconhecerem a importância do profissional farmacêutico e seus serviços prestados em consultório.

\section{REVISÃO DA LITERATURA}

\section{HISTÓRICO E EVOLUÇÃO DA ATENÇÃO FARMACÊUTICA}

Foi criado o decreto no 74.170/1974 (Brasil, 1974), que Regulamenta a Lei número 5.991/1973 (Brasil, 1973), que dispõe sobre o controle sanitário do comércio de drogas, medicamentos, insumos farmacêuticos e correlatos. Onde o Art $57 \mathrm{diz}$ que é vedado utilizar qualquer dependência da farmácia ou da drogaria como consultório, ou em outro fim diverso do licenciamento (nessa época consultório remetia apenas a consultórios médico, hoje consultórios é polisemantico, tem vários significados e abrange várias profissões) (CRF, 20II). 
Conhecidos como os pais dos "cuidados farmacêuticos, Hepler e Strand, definem esses cuidados como sendo toda a prestação de cuidados que envolve a dispensa dos medicamentos ao doente, com o principal objetivo de obter resultados no tratamento que melhorem a sua qualidade de vida" (MARTINS; COSTA; CARAMONA, 2013).

Após 20 anos dessa definição a Pharmaceutical Care Network Europe (PCNE), que é a Rede de Cuidados Farmacêuticos da Europa (PCNE), foi estabelecida em 1994 por um número de pesquisadores europeus de assistência farmacêutica. Tornou-se uma associação oficial (sob a lei holandesa) em 2004. E segunda ela, em consenso sobre a Atenção Farmacêutica: "Assistência Farmacêutica é a contribuição do farmacêutico para o cuidado dos indivíduos, a fim de otimizar o uso de medicamentos e melhorar os resultados de saúde" (ALLEMANN et al., 2014).

No Brasil, de acordo com a Resolução de no 585/2013 (Brasil, 2013) pelo Conselho Federal de Farmácia (CFF), que regulamenta as atribuições clínicas dos farmacêuticos, que atualmente na Farmácia Clínica incorpora todos os níveis de atenção à saúde, o que abre possibilidade de ser desenvolvida em vários ambientes como hospitais, unidade de atenção primária à saúde, farmácias comunitárias, instituições de longa permanência e domicílios, ambulatórios, entre outros. Sendo definida, de maneira mais ampla, como:

\footnotetext{
Área da farmácia, voltada à ciência e à prática do uso racional de medicamentos, na qual os farmacêuticos prestam cuidado ao paciente, de forma a otimizar a farmacoterapia, promover saúde e bem-estar, e prevenir doenças (CFF, 2016, p I86).
}

Nesse cenário clínico farmacêutico, essa RDC 585/CFF de 2013, que vai regulamentar as atribuições clínicas do farmacêutico que, por definição, constituem os direitos e responsabilidades desse profissional no que concerne a sua área de atuação.

A Resolução CFF nº 586/2013 (Brasil, 2013) encerra a concepção de prescrição como a ação de recomendar algo ao paciente. Tal recomendação pode incluir a seleção de opção terapêutica, a oferta de serviços farmacêuticos, ou o encaminhamento a outros profissionais ou serviços de saúde. Vale ressaltar que concepções de prescrição farmacêutica encontram-se fragmentadas na legislação vigente, tanto sanitária como profissional. A Resolução CFF n⿳0 586/2013 inova ao considerar a prescrição como uma atribuição clínica do farmacêutico, definir sua 
natureza, especificar e ampliar o seu escopo para além do produto e descrever seu processo na perspectiva das boas práticas, estabelecendo seus limites e a necessidade de documentar e avaliar as atividades de prescrição. $\mathrm{O} C F F$, ao regular a prescrição farmacêutica, o faz em consonância com as tendências de maior integração da profissão farmacêutica com as demais profissões da área da saúde, reforça a sua missão de zelar pelo bem-estar da população e de propiciar a valorização técnicocientífica e ética do farmacêutico (CFF, 2013).

É na prática desses serviços que vai haver a necessidade do farmacêutico possuir um método clínico completo de atendimento aos pacientes, um sistema de registro confiável que abarque suas responsabilidades profissionais e seu amplo conhecimento (CORRER, OTUKI, 2013).

Atualmente, os principais métodos de seguimento farmacoterapêutico utilizados na literatura internacional e no Brasil para a documentação desse cuidado são: SOAP; PWDT; TOM; Dáder. Sendo o método de SOAP o mais usado para o ciclo de atendimento (CORRER; NOBLAT; CASTRO, 2012).

SOAP (Subjetivo, Objetivo, Avaliação e Plano): Cada ponto desse método refere-se a uma parte do processo que se inicia com primeiro contato com o paciente para que ocorra o registro das informações sobre uso de medicamentos e a relação com a enfermidade; Já as informações objetivas coletadas vão estar em concordância com sinais vitais, resultados de exames e testes laboratoriais. A avaliação é a união dos dados onde deve-se relacionar com os medicamentos e intervenções, por final tem o plano onde tudo será estabelecido para instaurar o cuidado, e dessa forma ocorre o ciclo do atendimento (HURLEY, 2004; ROVERS et al., 2003).

Dessa forma, o Conselho Federal de Farmácia, define a consulta farmacêutica como o "Episódio de contato entre o farmacêutico e o paciente, com a finalidade de obter os melhores resultados com a farmacoterapia, promover o uso racional de medicamentos e de outras tecnologias em saúde. Objetiva, ainda, a promoção, proteção e recuperação da saúde, a prevenção de doenças e de outras condições, por meio da execução de serviços e de procedimentos farmacêuticos” (CFF, 2016).

\section{CONSULTÓRIO FARMACÊUTICO}

O Consultório Farmacêutico é um local onde o farmacêutico realiza com privacidade a consulta farmacêutica, a qual é destinada ao atendimento de pacientes, familiares e cuidadores (BRASIL, 2013). 
Dentro deste espaço, durante o atendimento, respeita os princípios éticos e profissionais, com o propósito de obter os melhores resultados com a farmacoterapia e com as orientações quanto ao uso racional de medicamentos e de outras tecnologias em saúde. Além disso, o consultório pode funcionar de modo autônomo ou como dependência de hospitais, ambulatórios, farmácias comunitárias, unidades multiprofissionais de atenção à saúde, instituições de longa permanência e demais serviços de saúde, no âmbito público e privado (OMS, 2013).

Nesse local são realizadas atribuições clínicas do farmacêutico relativas ao cuidado à saúde, nos âmbitos individual e coletivo, além de atribuições relacionadas à comunicação e educação em saúde, e à gestão da prática, produção e aplicação do conhecimento (CFF, 2016).

Há orientações quanto a medicação (posologia, dosagem e horários), sobre interações e desconfortos inerentes ao medicamento e a doença, e avaliação da prescrição médica. Na avaliação da prescrição médica entende-se como auxílio na interpretação da mesma, garantindo conhecimento sobre função dos medicamentos bem como doenças diagnosticadas e não diagnosticadas, colaborando assim como médico e outros profissionais da saúde em diversos aspectos do tratamento (LOPES, 2017).

FIGURA r: Exemplo de atendimento em consultório farmacêutico.

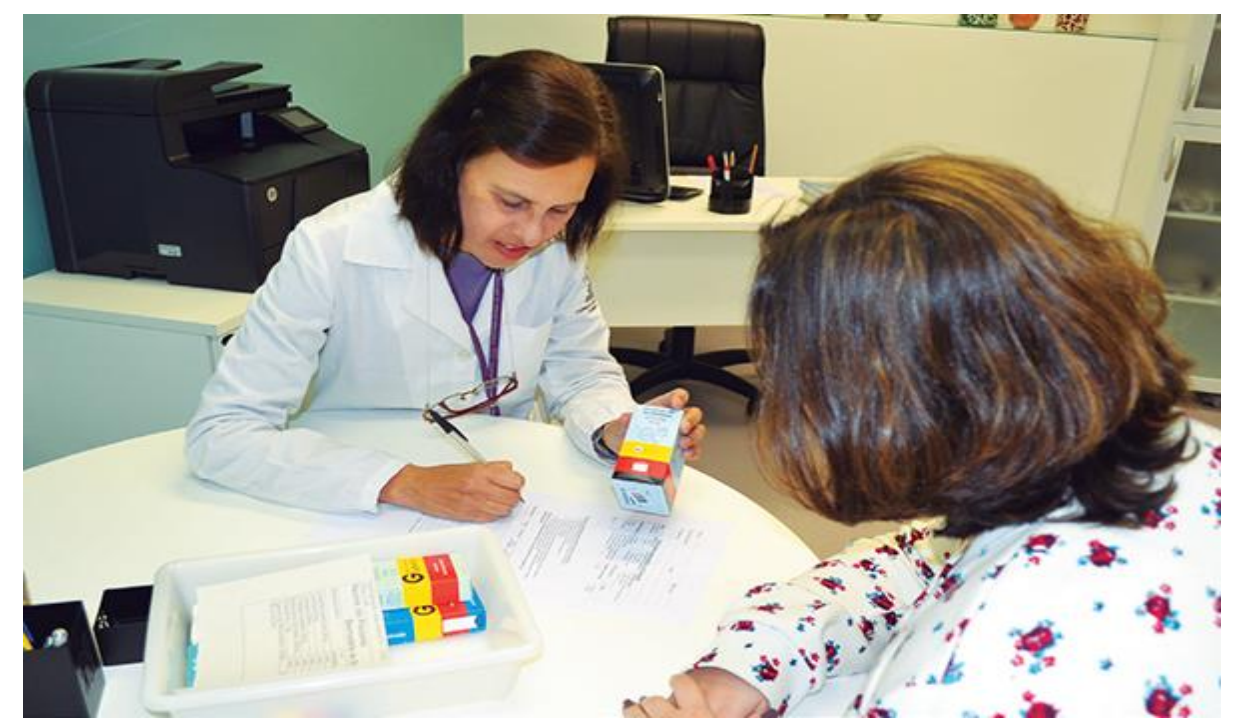

FONTE: CRFSP, 2017

A implantação de consultas farmacêuticas vem proporcionando a realização de um atendimento mais humanizado ao paciente, o que consequentemente permite 
gerar valorização profissional, maior adesão e eficácia aos tratamentos recomendados (LOPES, 2017).

Um farmacêutico atuante consegue, de maneira efetiva, aumentar a adesão ao tratamento, diminuir os custos dos sistemas de saúde, monitorar a presença de possíveis reações adversas aos medicamentos (RAMs) e interações medicamentosas, assim como reestabelecer como um todo, a qualidade de vida dos pacientes por ele atendidos (BISSO, M. P., 2or6; FERRACINI, F. T. et al., 2014; STORPITIS, S. et al., 2008).

\section{SERVIÇOS FARMACÊUTICOS}

As pesquisas direcionadas aos serviços farmacêuticos dos consultórios farmacêuticos e assistência farmacêutica teve como resultado que os serviços oferecidos pelos consultórios farmacêuticos são em geral: checkup de Pressão Arterial, de Glicemia, Colesterol e Risco Cardiovascular. Aplicação de injetáveis e Vacinação (+5o vacinas). Perfuração de Lóbulo, Medidas Corporais, Bioimpedância (o mesmo que medira quantidade de gordura e músculo no corpo). Controle do Tabagismo, da Asma. Avaliação de Saúde Mental, da Gestante. Testes Laboratoriais Remotos (+20 exames) (CLINICARX, 2020).

O serviço de vacinação não poderá ser oferecido por qualquer estabelecimento, será necessário atender às exigências e estar inscrito no Cadastro Nacional dos Estabelecimentos de Saúde (CNES), e deverá disponibilizar o calendário nacional de vacinação e os tipos de medicamentos disponíveis aos clientes (ADRENALINE, 2018; CRF SP, 2018).

Segundo à ADRENALINE (2020) os serviços que são oferecidos nos consultórios farmacêuticos são:

- Orientação do paciente sobre como usar medicamentos prescritos;

- Avaliação do conjunto de medicamentos usados pelo paciente quanto à dosagem, horário de consumo e possíveis interações;

- Comunicação com outros profissionais da saúde que atendam o paciente para emitir parecer farmacêutico e discutir tratamentos de forma integrada;

- Encaminhamento de paciente a outros profissionais de saúde;

- Conversa com paciente sobre sintomas e evolução da doença; 
- Caso necessário, pedido de exames laboratoriais e realização de procedimentos: como medidas de pressão e temperatura;

- Registro de ações em prontuário do paciente;

- Prescrição de medicamentos isentos de prescrição.

Vê-se que são diversos os serviços que podem ser oferecidos no consultório farmacêutico, para atendimento das necessidades de saúde dos pacientes e da população. Além de todos aqui vistos, ainda podem ressaltar aqueles que estão relacionados aos medicamentos como conciliação de medicamentos, revisão da farmacoterapia, monitorização terapêutica de medicamentos e acompanhamento farmacoterapêutico (HILAB, 2019).

Lembrando que todo serviço oferecido nestes consultórios requer a entrega ao paciente da Declaração de Serviço Farmacêutico, em cumprimento ao Artigo 8I, Seção III, da RDC-ANVISA nº 44/2009 (Brasil, 2009). Se for o caso de serviço não gratuito, a deve, o paciente, receber o recibo de serviços, de acordo com a legislação vigente em cada Estado da federação (VIEIRA E QUINTELLA, 2020).

Inclusive um grande desafio do profissional farmacêutico é efetuar o acompanhamento farmacoterapêutico, tempo esse necessário, para as anotações, as orientações e prevenir problemas alistados aos medicamentos (DE PAULA, 2019).

Muitos são os serviços que podem ser oferecidos no consultório farmacêutico, porém é fator determinante o tamanho, os aparelhos, enfim a estrutura montada.

FIGURA 2: Farmacêutico aferindo a pressão arterial.

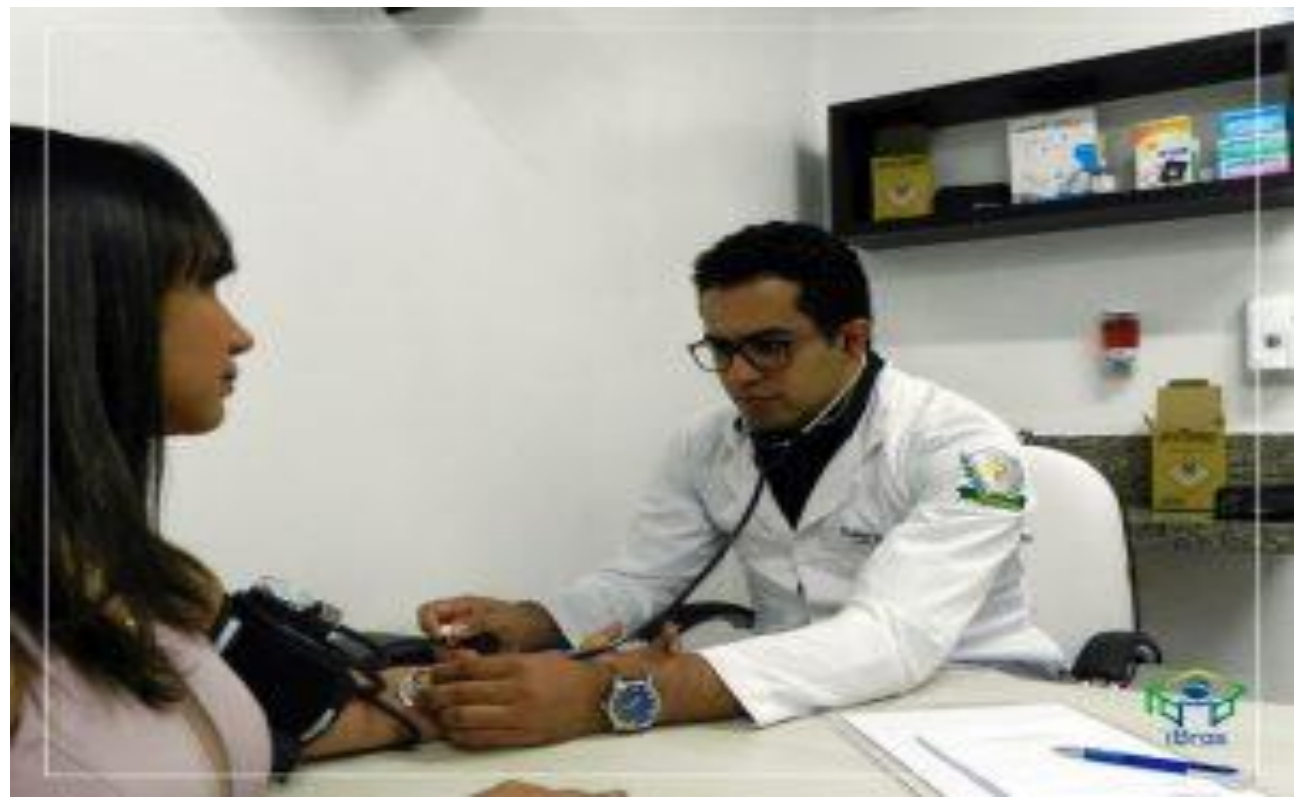

FONTE: IBRAS, 2018. 


\section{INTERVENÇÕES FARMACÊUTICAS EM CONSULTÓRIO}

Segundo ZUBIOLI (2013), Intervenções Farmacêuticas são todas ações da qual o farmacêutico participa ativamente como nas tomadas de decisão na terapia dos pacientes e também na avaliação dos resultados. Sendo uma etapa do Seguimento Farmacoterapêutico na Atenção Farmacêutica e na Farmácia Clínica, é a parte mais importante do acompanhamento farmacoterapêutico, onde ocorre a orientação do paciente e a atuação efetiva do profissional farmacêutico, visando identificar e prevenir problemas relacionados aos medicamentos, aumentando a efetividade e diminuindo os riscos da farmacoterapia.

Também pode ser considerado um ato planejado, documentando e realizado junto ao usuário e profissionais de saúde que visam resolver ou prevenir problemas que interferem ou podem interferir na farmacoterapia, sendo parte integrante do processo de acompanhamento/seguimento farmacoterapêutico. As intervenções podem ser entre Farmacêutico-Paciente, quando está relacionado ao uso do medicamento e/ou entre Farmacêutico-Doente-Médico, quando a farmacoterapia escolhida não alcança os efeitos esperados, ou se trata de um problema de saúde que necessite de diagnóstico médico (STURARO, 2013).

Considera-se uma intervenção aceite, quando o doente (no caso de intervenções Farmacêutico - Doente) ou o médico (no caso de intervenções Farmacêutico-Doente-Médico) modificam o uso do medicamento (dose, posologia, troca de medicamento) para tratar o problema em consequência da intervenção do Farmacêutico. O problema de saúde está resolvido quando em consequência da intervenção do Farmacêutico desaparece o motivo da mesma (MACHUCA, M; FERNANDEZ-LLIMOS, F; FAUS, 2013).

Estudos demonstram a importância das intervenções realizadas por farmacêuticos em estabelecimentos de saúde, como demonstram CARDINAL e FERNANDES (2013) onde mais de 99\% das intervenções farmacêuticas foram aceitas pelo corpo clínico (WEBER et al., 2013), o estudo mostrou que as intervenções farmacêuticas realizada no tratamento de antibióticos foi capaz de reduzir a duração 
da terapia, menor custo e maior efetividade e segurança para os pacientes. Um estudo de revisão realizada por ROMANO-LIEBER et al., (2013) em intervenções farmacêuticas em pacientes idosos foi capaz de otimizar o tratamento, reduzir custos, reduzir o número de problemas de prescrições, aumentar a adesão terapêutica, e controlar a possibilidade de eventos adversos.

\section{IMPLANTAÇÃO DE CONSULTÓRIO FARMACÊUTICO}

O consultório farmacêutico ao qual é referido trata-se de um espaço reservado dentro da drogaria e ou farmácia para que o farmacêutico possa fazer a computação do conjunto dos remédios que o enfermo está ingerindo, onde possa fazer as orientações e ouvir os pacientes com relação à forma de tomar os medicamentos e seu processo de mudança e adaptação clínica, entre outros procedimentos (LENHARO, 2017; FRANÇA, 2018).

Existem duas exigências iniciais para se montar um consultório farmacêutico, que são: a formação do empreendedor e a capacitação do mesmo. Com relação a formação, esse deve ser graduado, no mínimo como bacharel em farmácia, por uma faculdade reconhecida pelo MEC (Ministério da Educação) e estar registrado no CRF (Conselho Regional de Farmácia) da Cidade em que morar. E com relação à capacitação, trata-se de uma formação clínica, pois este necessitará de capacidade, conhecimentos e competências clínicas, assim como de gestão de serviços de saúde (LEONARDI, 2019).

Já a implementação efetiva e sustentável dos serviços farmacêuticos vai exigir um prosseguimento de fases que devem ser respeitadas e cumpridas, que inserem componentes do plano de negócio e de marketing, ainda diversos conhecimentos e habilidades consideradas novos, diante da formação de farmacêutico (NASCIMENTO, 2ог; ANGONESI, RENNÓ, 2оII).

A quantidade de Consultórios Farmacêuticos fica por conta do Censo Demográfico Farmacêutico, que pesquisou em 2017 e segundo as preliminares realizadas pelo Instituto de Ciência, Tecnologia e Qualidade (ICTQ), existiam I.453 consultórios farmacêuticos em todo o país. (CFF, 2017; CRF/PR 2018). 
QUADRO I: Implantação dos serviços farmacêuticos:

\begin{tabular}{|l|l|}
\hline $1^{\circ}$ passo: o começo & Que são as etapas de implementação \\
\hline $2^{\circ}$ passo: o produto & $\begin{array}{l}\text { Tornar possível os serviços que atendam a } \\
\text { população. }\end{array}$ \\
\hline $\begin{array}{l}3^{\circ} \text { passo: estrutura } \\
\text { fisica do consultório }\end{array}$ & $\begin{array}{l}\text { A estrutura fisica do consultório deve-se } \\
\text { adequar à legislação vigente. }\end{array}$ \\
\hline $4^{\circ}$ passo: processos & Tem que se agregarem valores ao serviço \\
\hline $5^{\circ}$ passo: evidências & $\begin{array}{l}\text { Ou como são chamados; os desafios do } \\
\text { trabalho oferecido pelo farmacêtico. }\end{array}$ \\
\hline $6^{\circ}$ passo: o preço & Devem ser calculados corretamente \\
\hline $7^{\circ}$ passo: promoção & Tem que ter programas promocionais \\
\hline $\begin{array}{l}8^{\circ} \text { passo: o } \\
\text { profissional }\end{array}$ & $\begin{array}{l}\text { (pessoas) cuidar bem das pessoas garante } \\
\text { o sucesso do consultório. }\end{array}$ \\
\hline $9^{\circ}$ passo: métricas & $\begin{array}{l}\text { O farmacêtico só pode melhorar aquilo } \\
\text { que mede e só pode medir aquilo que } \\
\text { registra e finalmente }\end{array}$ \\
\hline $\begin{array}{l}10^{\circ} \text { passo: o } \\
\text { software }\end{array}$ & $\begin{array}{l}\text { Que deve ser um que faça uma análise dos } \\
\text { dados de forma rápida e eficiente }\end{array}$ \\
\hline
\end{tabular}

FONTE: Nascimento (2018)

Segundo a Assistência Farmacêutica Avançada, consultórios farmacêuticos vêm crescendo de 2017 até 2019, sendo apontados mais de três mil estabelecimentos com consultórios farmacêuticos e os serviços cresceram tanto que alcançaram cinco milhões de atendimentos realizados no triênio 2017 a 2019 (ABRAFARMA, 2019).

Esse recente modelo de consultório, está em pleno crescimento, em 2018 já tinham sido implantados em "trezentos e cinquenta Municípios Brasileiros, identificando oitenta farmácias no Estado de São Paulo e quinhentas e oitenta e cinco na região Sudeste.” (RF, 2018). “A Associação Brasileira de Redes de Farmácias e Drogarias (Abrafarma) apontam que as salas de serviços farmacêuticos já estão presentes em 5 redes de farmácias: Pague Menos, Drogão Super, Farmaponte, Drogal e Raia Drogasil (RD).” (RF, 2018).

\section{CONCLUSÃO}

A Atenção Farmacêutica é uma prática farmacêutica importante para o sistema de saúde brasileiro, já que pode beneficiar o paciente no uso racional e eficaz de medicamentos.

Com o passar dos anos, além do aumento do número de intervenções farmacêuticas houve aumento na aceitabilidade associado a mudança no perfil de intervenções realizadas. $O$ aumento na aceitabilidade pode estar relacionado ao 
entendimento do papel do farmacêutico como profissional importante no sucesso da terapia medicamentosa e na segurança do uso dos medicamentos.

O farmacêutico, mais do que nunca, tem um papel importante junto à construção de um novo modelo de atenção à saúde, onde ele possa estar inserido como profissional do medicamento, atuando como referência na orientação, cumprimento, acompanhamento e monitoramento da terapia farmacológica.

Porém, algumas deficiências precisam ser corrigidas, por exemplo, em relação à formação e capacitação do farmacêutico, que deve ser criticamente voltada para um novo perfil profissional. O farmacêutico precisa ser formado para ser um profissional da saúde que pense criticamente a sua prática, e não mais um técnico em medicamentos.

\section{REFERÊNCIAS}

ABRAFARMA. Associação Brasileira de Redes de Farmácias e Drogarias. 7 tendências dos serviços farmacêuticos para 2020. 2019. Disponível em https://www.assistenciafarmaceutica.far.br/7-tendencias-dos-servicosarmaceuticos-para-2020/. Acesso em: 24 de setembro de 2021.

ADRENALINE. Vacinas agora podem ser dadas em farmácias de todo o Brasil. 2018. Disponível em https://adrenaline.com.br/forum/threads/disputa-entre-medicos-xfarmaceuticos-x-biomedicos-vai-parar-na-justica.630843/. Acesso em: is de setembro de 2021.

ALLEMANN, S. S. et al. pharmaceutical care: the PCNE definition 2013. International journal of clinical pharmacy.,v. 36, n. 3, p. 544-555, 2014.

ANGONESI, Daniela.; RENNÓ, Marcela Unes Pereira. Dispensação farmacêutica: proposta de um modelo para a prática. Faculdade de Ciências Biológicas e da Saúde. Belo Horizonte - Minas Gerais: 20Ir.

BISSO, M. P. Farmácia Clínica \& Atenção Farmacêutica. 3. ed. Barueri, São Paulo. Ed. Manole. 2016.

BRASIL. Conselho Federal de Farmácia. Resolução № 585 de 29 de agosto de 2013. Regulamenta as atribuições clínicas do farmacêutico e dá outras providências. 2013. 
Disponível em: http://www.cff.org.br/userfiles/file/resolucoes/585.pdf>. Acesso em: I4 de setembro de 202I.

CFF - Resolução no 586, de 29 de agosto de 2013. Regula a prescrição farmacêutica e dá outras providências. Brasília: Diário Oficial da União; 2013. Seção I, pp. 136-8 p

CFF - Serviços farmacêuticos diretamente destinados ao paciente, à família e à comunidade: contextualização e arcabouço conceitual / Conselho Federal de Farmácia - Brasília: Conselho Federal de Farmácia - CFF, 2016.

CRF/PR - Conselho Regional de Farmácia do Paraná. Manual da legislação, decreto no 74.170. Paraná: 2011. Disponível em: https://www.crfpr.org.br/uploads/noticia/7848/Manual_de_Lesgislao_20II.pdf Acesso em: o7 de outubro de 2021.

CRF/PR - Conselho Regional de Farmácia do Paraná. Consultório e Serviços Farmacêuticos. Paraná: 2018.

CLINICARX - Plataforma Digital de Saúde. Evite perder tempo e dinheiro apostando em modelos de serviços que não funcionam. São Paulo: 2020.

CORRER, C. J.; NOBlAT, L. A. C. B.; CASTRO, M. S. Gestão da Assistência Farmacêutica:módulo optativo: Unidade 3: modelos de seguimento farmacoterapêutico. 2012.

CORRER, C. J.; OTUKI, M. F. A Prática Farmacêutica na Farmácia Comunitária. Porto Alegre: Artmed, 2013.

DE PAUlA, C.C.; OIVEIRA, G. F. de.; LAMARE, C. A. V. de; SHIMOYA, W. Importância das intervenções farmacêuticas na prática clínica. Cap. I -Livro: Farmácia clínica e atenção farmacêutica. Atena Editora, 2019.

FIGURA r: CRFSP, 2017. Disponível em: http://portal.crfsp.org.br/geral/revista13I/9155-revista-do-farmaceutico-13I-portaria-cvs Acesso em: 25 de setembro de 2021. 
FIGURA 2: IBRAS, 2018. Disponível em: http://blogibras.com.br/5-desafios-quefarmaceutico-deve-superar-para-ter-seu-proprio-consultorio/ Acesso em: 25 de setembro de 202I.

FRANÇA, Ana Luce. Consultório farmacêutico: uma nova perspectiva de mercado. UNIDESC Centro Universitário. 2018.

HEPLER, C.D.; STRAND, L.M. Opportunities andresponsibilities in pharmaceutical care. Am. J. Hosp.Pharm., v.47, n.3, p.533-543, 1990.

HILAB, Marketing. Serviços farmacêuticos: o que são e por onde começar? (2019). Disponível em https://hilab.com.br/blog/servicos-farmaceuticos-o-que-sao/. Acesso em: 16 de setembro de 2021.

HURLEY, S.C. A method of documenting pharmaceutical care utilizing pharmaceutical diagnosis. College of Pharmacy, Idaho State University, 2004.

LENHARO, Mariana. Consultórios farmacêuticos: entenda como é atendimento que vem sendo oferecido por farmácias. Entrevista com farmacêutico Ronaldo Ribeiro. Drogaria com atendimento clínico na Mooca, em São Paulo. 2017.

LEONARDI, Egle. 16 Passos para Montar um Consultório Farmacêutico. ICTQ Instituto de Ciência, Tecnologia e Qualidade. Anapolis - GO: 2019

LOPES, Denise Aparecida Moreira Gollner. ATENÇÃO FARMACÊUTICA E CONSULTÓRIOS FARMACÊUTICOS. Revista Acadêmica Oswaldo Cruz, ano 4, n.16 outubro-dezembro 2017 ISSN 2357-81873 (versão on-line). Disponível em: http://revista.oswaldocruz.br/Artigos. Acesso em: I4 de setembro de 2021.

MACHUCA, M; FERNANDEZ-LlimOS, F; FAUS, M.J. Método Dadér. Guia de Seguimento Farmacoterapêutico, 2013. Tradução, 2013. Disponível em: http://www.farmaceuticogaucho.pro.br/metodo\%2odader\%2opdf.pdf. Acesso em: 24 de setembro de 202I. 
MARTINS S, COSTA FA, CARAMONA M. Implementação de Cuidados Farmacêuticos em Portugal, Seis Anos Depois. Rev Port Farmacoter, v. 5, p. 255-263, 2013.

NASCIMENTO, Carlos. Dez passos para implantar um consultório farmacêutico. Revista do Farmacêutico no 133 . São Paulo: 2018.

RF -. Consultórios farmacêuticos e modelos focados em assistência farmacêutica começam a transformar a Farmácia brasileira. Vol. 133. Publicação do Conselho Regional de Farmácia do Estado de São Paulo: 2018.

ROMANO-LIEBER, N. S.; TEIXEIRA, J. J. V.; FARHAT, F. C. L. G.; et al. Revisão dos estudos de intervenção do farmacêutico no uso de medicamentos por pacientes idosos. Caderno de Saúde Pública. v. I8, n.6, p. I499-1507, 2013.

ROVERS, J. P., et al. A practical guide to pharmaceutical care. Washington: American Pharmaceutical Association, p. 316, 2003.

STURARO, D. S. A importância do acompanhamento farmacoterapêutico em

pacientes onco-hematológicos. Revista Brasileira de Hematologia e Hemoterapia, n.31, v.3, p.124, 2013.

VIEIRA, Gabriele Brasil Barbiere; QUINTELLA, Cesar Mathias. Manual do Consultório Farmacêutico. Rio de Janeiro: 2020.

WEBER, A et al. Interventions by Clinical Pharmacists on Cirurgical Wards Impact on AntibioticTherapy. Zentralbl Chir, n.136, p.66-73, 2013.

ZUBIOLI, A. O farmacêutico e a auto-medicação responsável. Pharmácia Brasileira. v. 3, n. 22, p. 23-26, 2013 . 